\title{
The effects of automated artifact removal algorithms on electroencephalography-based Alzheimer's disease diagnosis
}

\author{
Raymundo Cassani ${ }^{1}$, Tiago H. Falk ${ }^{1 *}$, Francisco J. Fraga ${ }^{1,2}$, Paulo A. M. Kanda ${ }^{3}$ and Renato Anghinah ${ }^{3}$ \\ 1 Institut National de la Recherche Scientifique, Centre Énergie, Matériaux, Télécommunications, University of Quebec, Montreal, QC, Canada \\ ${ }^{2}$ Engineering, Modelling and Applied Social Sciences Center, Universidade Federal do ABC, São Paulo, Brazil \\ ${ }^{3}$ Reference Center of Behavioural Disturbances and Dementia, School of Medicine, Universidade de São Paulo, São Paulo, Brazil
}

\section{Edited by:}

Davide V. Moretti, IRCCS San

Giovanni di Dio Fatebenefratelli, Italy

Reviewed by:

Xiaoli Li, Beijing Normal University,

China

Umberto Melia, Universitat

Politecnica de Catalunya, Spain

*Correspondence:

Tiago H. Falk, Institut National de la Recherche Scientifique, Centre

Energie, Matériaux

Télécommunications, University of Quebec 800, Rue de la Gauchetire Ouest, Suite 6900 Montreal, OC

H5A-1K6, Canada

e-mail: falk@emt.inrs.ca
Over the last decade, electroencephalography (EEG) has emerged as a reliable tool for the diagnosis of cortical disorders such as Alzheimer's disease (AD). EEG signals, however, are susceptible to several artifacts, such as ocular, muscular, movement, and environmental. To overcome this limitation, existing diagnostic systems commonly depend on experienced clinicians to manually select artifact-free epochs from the collected multi-channel EEG data. Manual selection, however, is a tedious and time-consuming process, rendering the diagnostic system "semi-automated." Notwithstanding, a number of EEG artifact removal algorithms have been proposed in the literature. The (dis)advantages of using such algorithms in automated AD diagnostic systems, however, have not been documented; this paper aims to fill this gap. Here, we investigate the effects of three state-of-the-art automated artifact removal (AAR) algorithms (both alone and in combination with each other) on $A D$ diagnostic systems based on four different classes of EEG features, namely, spectral, amplitude modulation rate of change, coherence, and phase. The three AAR algorithms tested are statistical artifact rejection (SAR), blind source separation based on second order blind identification and canonical correlation analysis (BSS-SOBI-CCA), and wavelet enhanced independent component analysis (wICA). Experimental results based on 20-channel resting-awake EEG data collected from 59 participants (20 patients with mild $A D, 15$ with moderate-to-severe $A D$, and 24 age-matched healthy controls) showed the wICA algorithm alone outperforming other enhancement algorithm combinations across three tasks: diagnosis (control vs. mild vs. moderate), early detection (control vs. mild), and disease progression (mild vs. moderate), thus opening the doors for fully-automated systems that can assist clinicians with early detection of $A D$, as well as disease severity progression assessment.

Keywords: Alzheimer's disease, automatic diagnosis, electroencephalogram, amplitude modulation, EEG artifacts, SVM

\section{INTRODUCTION}

Alzheimer's disease (AD) is a chronic neuro-degenerative disorder that has recently been ranked as the third most expensive disease and the sixth leading cause of death in the United States (Leifer, 2003; Alzheimer Association, 2013). In 2012, the World Health Organization (WHO) stated that between $60-70 \%$ of dementia cases around the world were due to $\mathrm{AD}$, making it the most common form of dementia. As such, it called for improved (early) diagnosis, as well as better care and support for patients, their families, and caregivers (WHO and Alzheimer's Disease International, 2012). With regards to the former, today diagnosis is commonly carried out using laboratory tests, medical history, mental status examinations, and more recently, neuroimaging tools such as functional magnetic resonance imaging (fMRI). These clinical assessment methods, however, commonly require experienced clinicians and lengthy sessions, thus can be regarded as non-specific and costly, as well as suffer from long wait times to access an fMRI scanner. In medium- and low-income countries, as well as in rural and remote regions (e.g., the Canadian Arctic), these limitations are further exacerbated, thus hindering the effectiveness of very early disease diagnosis (Sarazin et al., 2012).

Driven by these limitations, quantitative electroencephalography (qEEG, henceforth referred to as "EEG") has emerged as a promising tool capable of assisting physicians in the diagnosis of AD (e.g., Jeong, 2004; Babiloni et al., 2010; Falk et al., 2012). Since the EEG signal reflects functional changes in the cerebral cortex, it can be used to reveal neuronal degeneration and functional impairment long before actual tissue loss can be detected by fMRI (Alzheimer Association, 2013). Over the last decade, several works have demonstrated a neuromodularity deficit with $\mathrm{AD}$ via EEG signal analysis (e.g., Jeong, 2004; Dauwels et al., 2011; Moretti et al., 2012). For example, apparent changes in the EEG power spectrum (e.g., slowing of the EEG) have been documented 
(Coben et al., 1983, 1985; Brenner et al., 1986; Giaquinto and Nolfe, 1986), as well as reduced spectral coherence between the left and right hemispheres (Leuchter et al., 1987; Besthorn et al., 1994; Dunkin et al., 1994; Sloan et al., 1994; Locatelli et al., 1998). Moreover, EEG signal complexity measures have shown decreased levels with $\mathrm{AD}$, likely due to the reduction in non-linear connections between cortical regions or even neuronal death (Jeong, 2004). More recently, EEG amplitude modulation analysis has also shown to be a powerful tool in EEG diagnosis (Falk et al., 2012; Fraga et al., 2013b). Many such measures have been shown to be related (Dauwels et al., 2011) and to provide diagnostic sensitivity and specificity in line with more complex neuroimaging techniques (Adeli et al., 2005).

Notwithstanding, EEG signals are inherently noisy and susceptible to blink, eye movement, heartbeats, and cranial muscle artifacts, all of which are detrimental to $\mathrm{AD}$ diagnosis performance. To overcome this limitation, the majority of the published works have resorted to using artifact-free EEG segments (called epochs) which have been selected by expert clinicians via meticulous visual inspection. Such dependence on human experts, however, hinders the benefits of automated low-cost analysis, as well as introduces possible human biases/errors (Daly et al., 2013). As an alternative, artifact removal algorithms could be employed. Artifact removal algorithms can be classified as 'semiautomated' or 'automated', depending on the need for human intervention, or not, respectively. Component-based methods, such as independent component analysis (ICA), can be regarded as semi-automated methods, as signal components associated with artifacts still need to be manually identified by humans and removed prior to signal reconstruction (Jung et al., 2000; James and Hesse, 2005). On the other hand, wavelet denoising (Zikov et al., 2002; Krishnaveni et al., 2006), blind source separation (De Clercq et al., 2006; Gómez-Herrero et al., 2006), or even simple feature averaging (Fraga et al., 2013b), are fully automated methods that do not require human intervention. Within the scope of EEG-based AD diagnosis, the potential benefits and drawbacks of using automated artifact removal (AAR) algorithms are still unknown. For example, certain algorithms may remove important neurological phenomena needed for accurate diagnosis. The aim of this paper is to fill this gap and explore the (dis)advantages of utilizing AAR for EEG-based AD diagnosis.

Here, three AAR algorithms have been selected after careful screening of the literature for available state-of-the-art methods applicable to our data. The first method, termed statistical artifact rejection (SAR), utilizes statistical characteristics of the signals to make accept/reject decisions over EEG epochs (Delorme et al., 2007). The second method belongs to the widely-used class of blind source separation (BSS) algorithms based on the autocorrelation of independent components (De Clercq et al., 2006; Gómez-Herrero et al., 2006). Lastly, a combined independent components analysis and wavelet denoising algorithm, termed wavelet enhanced ICA (wICA), is used which applies a wavelet thresholding algorithm to replace the human intervention step required with ICA (Castellanos and Makarov, 2006). The three algorithms are tested alone and in combination with each other, as well as in combination with the simple feature averaging approach described by Fraga et al. (2013b). The AAR algorithms are applied to raw EEG data collected from 59 participants (20 patients with mild AD, 15 with moderate-to-severe $\mathrm{AD}$, and 24 age-matched healthy controls). Their effects on four classes of EEG features, namely spectral-, coherence-, phase-, and amplitude modulation-based features are tested and compared to a gold-standard method, which relies on expert human inspection of artifact-free epochs. The ultimate goal of the present paper is to describe the best AAR-feature set combination, thus resulting in a reliable system that can be used to assist clinicians in diagnosis and very early detection of $\mathrm{AD}$, as well to monitor disease progression.

\section{MATERIALS AND METHODS 2.1. PARTICIPANTS}

Fifty-nine participants were recruited from the Behavioral and Cognitive Neurology Unit of the Department of Neurology and the Reference Center for Cognitive Disorders at the Hospital das Clinicas in São Paulo, Brazil (Kanda et al., 2013). AD diagnosis was made by experienced neurologists according to NINCDSADRDA criteria (McKhann et al., 1984) and classified based on the Brazilian version of the Mini-Mental State Examination (MMSE) (Brucki et al., 2003). Participants were divided in three groups. The first group $(N)$ consisted of 24 cognitively healthy controls ( 12 males; mean age 66.3 years, $8.8 s d$ ); the second group $(A D 1)$ comprised 20 mild-AD patients ( 9 males, mean age 74.8 years, $6.3 s d$ ); the third group (AD2) consisted of 15 patients with moderate-to-severe $\mathrm{AD}$ symptoms ( 6 males; mean age 75 years, $11.8 s d$ ). Inclusion criteria for the $N$ group included a CDR score $=0$ and MMSE score $\geq 25$ (mean 28.5, $1.7 s d$ ), as well as no indication of functional cognitive decline. Inclusion criteria for the $A D 1$ group, in turn, included $0.5 \leq \mathrm{CDR} \leq 1$ and $\mathrm{MMSE} \leq 24$ (mean 19.2, $5.2 s d$ ); lastly, inclusion criteria for the AD2 group were $\mathrm{CDR}$ score $=2$ and MMSE $\leq 20$ (mean 12.8, $5 s d$ ). For inclusion to the two $\mathrm{AD}$ groups, an additional criterion used was the presence of functional and cognitive decline over the previous 12 months based on detailed interviews with knowledgeable informants. Patients from the AD cohorts were also screened for diabetes mellitus, kidney disease, thyroid disease, alcoholism, liver disease, lung disease or vitamin B12 deficiency, as these can also cause cognitive decline. Ethics approval was obtained from the Research Ethics Office and participants consented to participate in the study.

\subsection{EEG DATA ACQUISITION AND PRE-PROCESSING}

Twenty-channel EEG signals were acquired with the participants awake, relaxed, and with their eyes closed for at least $8 \mathrm{~min}$. The Braintech 3.0 instrumentation (EMSA Equipamentos Médicos INC., Brazil) was used with 12-bit resolution and $200 \mathrm{~Hz}$ sample rate parameters. Impedance was maintained below $10 \mathrm{k} \Omega$ and scalp electrodes were placed according to the international 10-20 system. Bi-auricular referential electrodes were attached, as recommended by the Brazilian Society of Clinical Neurophysiology and the American EEG Society. An infinite impulse response low-pass elliptic filter with a zero at $60 \mathrm{~Hz}$ was applied to eliminate power grid interference. Moreover, based on evidence of an interhemispheric disconnection with AD (Jeong, 2004; Trambaiolli et al., 2011b,c; Falk et al., 2012; Fraga et al., 2013b), 
we also explore the use of virtual interhemispheric bipolar signals. Interhemispheric bipolar signals refer to the electric potential difference measured between a pair of electrodes symmetrically located in each hemisphere. Moreover, the term "virtual" is used because these signals are mathematically computed as the difference of two recorded unipolar signals rather than directly recorded from the scalp (Nunez, 2006). The eight virtual bipolar signals explored in this work were the interhemispheric signals Fp1-Fp2, F7-F8, F3-F4, T3-T4, C3-C4, T5-T6, P3-P4, and O1-O2.

Unprocessed signals (both per-electrode and bipolar) constitute what will, henceforth, be referred to as the "raw" EEG. The enhanced signals, in turn, will constitute the raw signals processed by the different AAR algorithms described in the next subsection. Lastly, the raw signals have also been visually inspected by two experienced clinicians to obtain several 8-s epochs free of eye blinking, drowsiness, muscle movements, or equipment-related artifacts. This manually-selected data will be used to develop a gold-standard diagnostic system with which the AAR algorithms will be benchmarked against.

\subsection{AUTOMATED ARTIFACT REMOVAL (AAR) ALGORITHMS}

As mentioned previously, three AAR algorithms are explored within this work and were chosen based on characteristics of our dataset; more specifically, on the electrode layout (international 10-20 system), relatively small number of electrodes (20), absence of electrooculographic (EOG) reference channels, and lack of data from alternate modalities (e.g., accelerometers or gyroscopes). In the subsections to follow, a brief summary of the three AAR algorithms is given, as well as a description of their implementations. References to literature with more detailed descriptions of the algorithms are provided, where appropriate, for the interested reader.

\subsubsection{Statistical artifact rejection (SAR)}

The SAR method utilizes thresholding on the statistical characteristics of the EEG signals to select epochs that appear to contain artifacts. The implementation of this method was done using the well-known EEGLAB toolbox for Matlab (Delorme and Makeig, 2004). The criteria used to reject epochs included finding: extreme values caused by gross artifacts and amplifier saturation (i.e., greater than $+/-100 \mu \mathrm{V}$ ), abnormally distributed data (i.e., 5 standard deviations from average kurtosis, suggesting peaky or flat distributions) and "improbable data" computed via an online probability-of-occurrence metric. The interested reader is referred to (Delorme et al., 2007) for more details on the SAR algorithm.

\subsubsection{Blind source separation (BSS)}

The BSS algorithm utilizes spatial filtering to remove ocular and muscular artifacts from EEG data without external references (e.g., EOG or accelerometer signals) (De Clercq et al., 2006; Gómez-Herrero et al., 2006). The basic principle behind BSS is to decompose the EEG signal into different spatial components and then reconstruct the signal based only on the non-artifactual spatial components, which have been found via a suitable automatic criterion. For ocular and muscular artifacts, the EEG signal is decomposed by the so-called second order blind identification (SOBI) and canonical correlation analysis (CCA) methods, respectively. In the SOBI technique (Belouchrani et al., 1997; Gómez-Herrero et al., 2006; Romero et al., 2008), second order statistics are used to find spatial components that have nonzero time-delayed autocorrelations and zero time-delayed crosscorrelations. Such approach has been shown to preserve more brain activity relative to other ocular artifact removal methods (Romero et al., 2008). In our simulations, a fractal dimensionbased criterion was used to decide which components to use for reconstruction, with the basic premise that EEG artifacts are characterized by higher fractal dimensions (Gómez-Herrero et al., 2006). With CCA, in turn, EEG data is expressed as a combination of maximally autocorrelated and mutually uncorrelated spatial components (De Clercq et al., 2006). Using CCA, spatial components with the lowest autocorrelation values are assumed to be related to muscular artifacts, as muscular activity has been shown to be of wider bandwidth than EEG, thus have more white noise-like properties (De Clercq et al., 2006). For this experiment, BSS AAR refers to the use of the SOBI technique, followed by CCA to remove ocular and muscular artifacts, respectively. The widely-utilized AAR plug-in for EEGLAB was used in our experiments with the following default parameters: for EOG removal, eigenratio $=10^{6}$, range $=2-4$, and the no-EOG reference option selected; for EMG removal, emg - psd - ratio $=10$, and femg $=$ 15. More details about these parameters and the plug-in can be found in (Gómez-Herrero, 2007). For illustration purposes, Figure 1 depicts a 10-s segment of raw (gray) EEG along with its BSS-processed (green) counterpart for four electrodes affected by eye artifacts: Fp1, Fp2, F7, and F8.

\subsubsection{Wavelet-enhanced independent components analysis (wICA)}

Wavelet analysis has been used in the past for EEG artifact detection (e.g., Achanccaray and Meggiolaro, 2008) and removal (e.g., Labate et al., 2011) and has recently been combined with ICA for improved artifact removal performance (Castellanos and Makarov, 2006; Akhtar et al., 2012). The so-called wavelet enhanced ICA, or wICA, applies a wavelet thresholding step to the demixed independent components in an attempt to recover any residual neural activity that may be present in components labeled as artifactual (Castellanos and Makarov, 2006). The wICA method can be summarized in five steps: (1) the EEG data is decomposed into independent components (IC); (2) the wavelet transform is applied to the ICs; (3) thresholding of the wavelet coefficients is performed to differentiate between neural and artifactual coefficients; (4) the inverse wavelet transform is applied to the thresholded coefficients, retrieving ICs with only neural activity; and lastly, (5) wavelet-corrected ICs are projected to obtain the artifact-free EEG data. A complete description, as well as a comparative analysis between ICA and wICA is given by Castellanos and Makarov (2006); improved performance and better preservation of EEG spectral and phase coherence properties with wICA are shown. In our experiments, the wICA toolbox described by Makarov (2012) was used with the following parameters: cleaning artifact tolerance $=1.25$ and an IC artifact detection threshold $=4$. Figure 1 also shows the 10-s noisy EEG segment processed by wICA (black). As can be seen from the highlighted areas, wICA suppresses eye blink/movement artifacts more efficiently than BSS. 


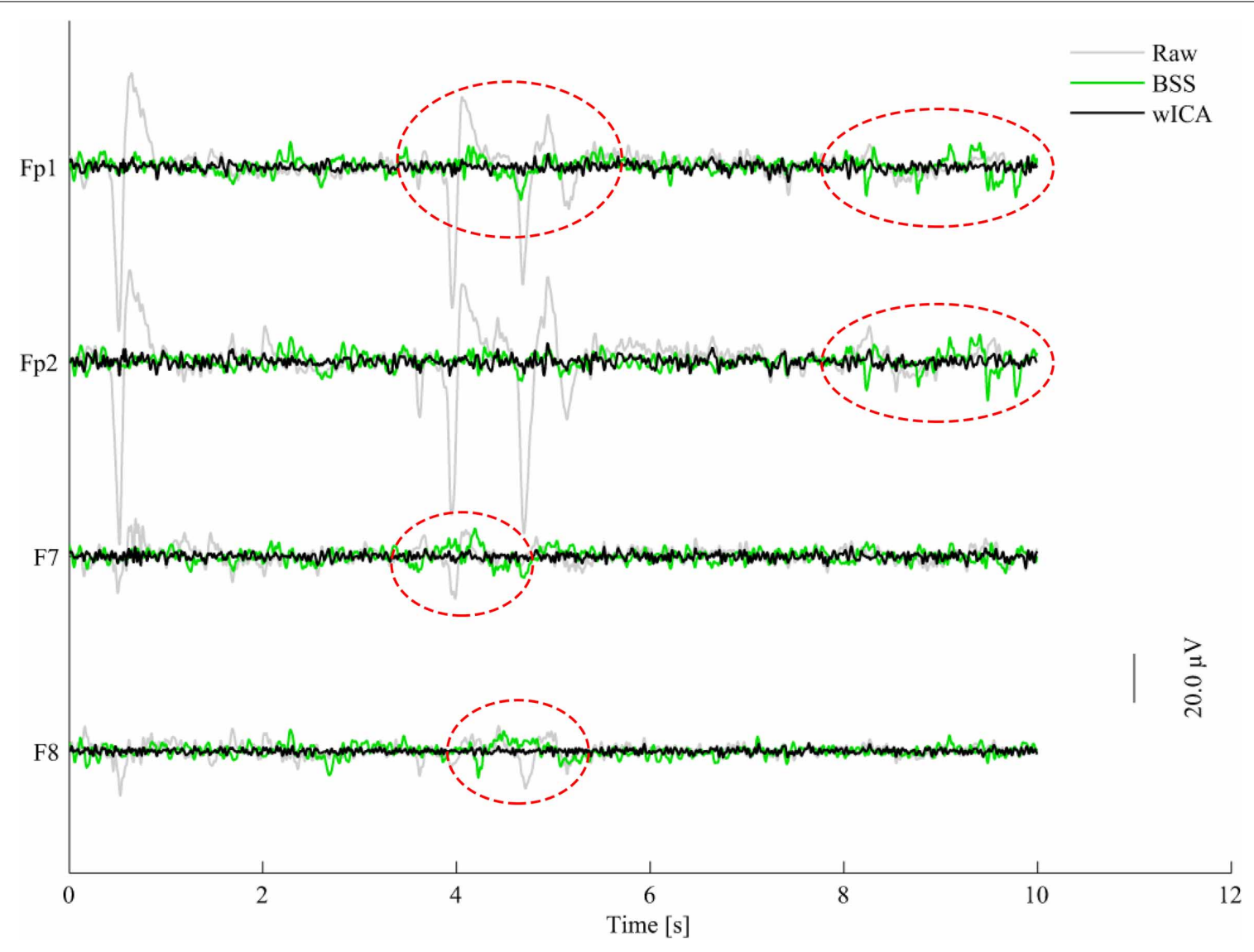

FIGURE 1 | Plots of raw (gray), BSS- (green), and wICA-processed (black) EEG segments for four channels corrupted by eye blinks and movement.

\subsubsection{AAR Algorithm Combination}

Here, we have tested the three above-mentioned AAR algorithms alone, as well as in cascade; more specifically, we have tested the SAR-BSS and SAR-wICA combinations. Overall, experimental results will be presented using the "raw" data (this will be henceforth refereed to as the "baseline"), the manually-selected artifactfree EEG data (henceforth referred to as the "gold-standard"), and the five "enhanced" EEG datasets (i.e., SAR, BSS, wICA, SARBSS, SAR-wICA). To maintain consistency with the gold-standard system, all datasets are segmented into several 8-s epochs.

\subsection{EEG FEATURE EXTRACTION AND PROCESSING}

Several EEG features have been proposed in the literature over the last decade and shown to accurately discriminate between healthy controls and AD patients. The effects of EEG artifacts on these features, however, are unknown, as are their effects on overall diagnostic performance. Here, we will pursue such an investigation and focus will be placed on four traditional EEG feature categories, namely, spectral power, magnitude square coherence, phase coherence/synchrony, and the recently-proposed EEG amplitude modulation rate-of-change. In the subsections to follow, a brief description of the features will be given. References to literature with more detailed descriptions of the features are provided, where appropriate, for the interested reader.

\subsubsection{EEG subband spectral power}

The pivotal process to quantify the frequency-domain properties of the EEG signal lies in the estimation of its power spectral density (PSD) function, which is commonly achieved via a discrete Fourier transform (Sörnmo and Laguna, 2005). As the name suggests, spectral power based features measure the power present in the five conventional EEG frequency bands: $0.1-4 \mathrm{~Hz}$ (delta), 4 $8 \mathrm{~Hz}$ (theta), $8-12 \mathrm{~Hz}$ (alpha), $12-30 \mathrm{~Hz}$ (beta) and, 30-100+ Hz (gamma) (Sörnmo and Laguna, 2005), with some studies further partitioning a band into low (e.g., alpha1: 8-10 Hz) and high (e.g., alpha2: $10-12 \mathrm{~Hz}$ ) parts. Several studies have shown that changes in EEG power spectra due to $\mathrm{AD}$ are reflected as an increase in delta and theta band powers, together with a decrease in alpha and beta band powers, thus suggesting a "slowing" of the EEG signal (Coben et al., 1983, 1985; Penttilä et al., 1985; Soininen et al., 1989; Czigler et al., 2008; Moretti et al., 2009; Babiloni et al., 2010). More recently, other features have been proposed, such as the subband spectral peaks (the most prominent peak inside a frequency band) (Raicher et al., 2008) and the ratio of different bands (e.g., theta/gamma by Moretti et al., 2009, 2011). In this experiment, we compute the so-called relative band power for the five bands for each of the 28 EEG signals (20 electrodes + 8 virtual bipolar signals). The relative band power corresponds to the power of an individual band normalized by the fullband EEG 
power. A total of $140(28 \times 5)$ spectral-based features are thus computed per epoch.

\subsubsection{Magnitude square and phase coherence}

The magnitude square coherence (MSC), frequently referred to as "coherence," is a measure of co-variance between two power spectra. In EEG studies, the MSC is used as a metric of synchrony in neural activity, which is an indicator of cortical connectivity (Thatcher et al., 1986; Locatelli et al., 1998; Srinivasan et al., 2007). Studies have shown reduced EEG coherence within all EEG subbands during AD (Thatcher et al., 1986; Besthorn et al., 1994; Knott et al., 2000; Adler et al., 2003). The computation of the MSC between signals $x(t)$ and $y(t)$ with $X(f)$ and $Y(f)$ spectra, respectively, for any given frequency band is defined as:

$$
\operatorname{MSC}(f)=\frac{\left|\left\langle X(f) Y^{*}(f)\right\rangle\right|^{2}}{|\langle X(f)\rangle||\langle Y(f)\rangle|},
$$

where $Y^{*}(f)$ is the complex conjugate of $Y(f),\langle\rangle$ corresponds to the average operator, and the numerator $\left\langle X(f) Y^{*}(f)\right\rangle$ corresponds to the cross-spectral density between signal $x(t)$ and $y(t)$, also called the complex coherence. The imaginary part of the complex coherence, also known as phase coherence, has also been proposed as metric to study brain interactions (Nolte et al., 2004). The phase coherence is given by:

$$
\phi(f)=\arg \left\langle X(f) Y^{*}(f)\right\rangle .
$$

In our experiments, we compute both metrics for each of the five EEG frequency bands. Following the recent evidence of an interhemispheric disconnection with AD (Jeong, 2004; Trambaiolli et al., 2011c,b; Falk et al., 2012; Fraga et al., 2013b), the magnitude square and phase coherence measures are computed only for the eight interhemispheric electrodes, namely: Fp1-Fp2, F7-F8, F3-F4, T3-T4, C3-C4, T5-T6, P3-P4, and O1-O2.

\subsubsection{Phase synchrony}

Global field synchrony (GFS) measures the phase synchrony in a given frequency (or frequency band) for a set of $N$ electrodes. It was first introduced to estimate the functional disorder within the brain for patients with schizophrenia (Koenig et al., 2001). Since AD has also been characterized by a loss of EEG synchrony resultant from the functional interhemispheric disconnection (Jeong, 2004), GFS has been explored as a diagnostic feature (Koenig et al., 2005; Park et al., 2008). Assuming $x_{i}(k)$, $i=1, \ldots, N$, are the EEG time-domain signals from electrode ' $i$ ' and $X_{i}(f)$ are their respective frequency responses (obtained via e.g., Fourier transform), the GFS feature is based on the distribution of the real $\left(X_{\mathbb{R}}(f)\right)$ and imaginary $\left(X_{\mathbb{I}}(f)\right)$ parts of the frequency-domain representation of all electrode signals. More specifically, it is computed as the difference between the two normalized eigenvalues of the $2 \times 2$ auto-correlation matrix between the vectors $X_{\mathbb{R}}(f)=\left[\operatorname{Re}\left(X_{1}(f)\right), \ldots, \operatorname{Re}\left(X_{N}(f)\right)\right]$ and $X_{\mathbb{I}}(f)=$ $\left[\operatorname{Img}\left(X_{1}(f)\right), \ldots, \operatorname{Im} g\left(X_{N}(f)\right)\right]$. More details about the GFS feature can be found in (Koenig et al., 2001). In our experiments, the GFS feature was computed over the 20 electrode signals for each of the five frequency bands, totaling five GFS features per EEG epoch.

\subsubsection{EEG amplitude modulation rate-of-change}

Amplitude modulation analysis has shown to be a valuable tool for bio-signal processing and analysis (Atlas and Shamma, 2003; Malyska et al., 2005; Falk and Chan, 2008; Falk et al., 2010). For AD analysis, it is particularly useful, as recent experimental evidence has suggested a neuromodulatory deficit with the disease (Moore and Cao, 2008; Laxton et al., 2010). Here, we utilize the EEG amplitude modulation rate-of-change features recently shown to accurately discriminate between different stages of $\mathrm{AD}$ (Trambaiolli et al., 2011b; Falk et al., 2012; Fraga et al., 2013a,b). In order to compute the features, three steps are required. First, the fullband EEG is frequency-decomposed into the five bands mentioned above. Second, a Hilbert transform is applied to extract the amplitude modulations of each band. Lastly, in order to characterize the dynamics of the amplitude modulations, a second frequency decomposition is performed on the band envelope signals. To characterize the cross-frequency interactions, this second decomposition utilizes five so-called "modulation bands" that have been designed to coincide with the frequency ranges of the five traditional subbands. To distinguish between frequency and modulation bands, the latter are referred to as $m$-delta, $m$ theta, $m$-alpha, $m$-beta and, m-gamma. The normalized energy in each frequency-modulation band is used as a feature. It is important to emphasize, however, that due to properties of the Hilbert transform [e.g., Bedrosian's theorem (Bedrosian, 1963)], not all frequency-modulation band combinations make sense. If we use the notation " $E$ (frequency band; modulation band)" to denote the normalized energy in a given frequency and modulation band, only the following scenarios are relevant: E(delta; $m$-delta), E(theta; $m$-delta, $m$-theta), E(alpha; $m$-delta, $m$-theta), $E$ (beta; $m$-delta, $m$-theta, $m$-alpha, $m$-beta) and, E(gamma; $m$ delta, $m$-theta, $m$-alpha, $m$-beta, $m$-gamma). In our experiments, these 14 features are computed for each of the 28 signals (20 electrodes +8 virtual bipolar signals). The interested reader is referred to (Trambaiolli et al., 2011b; Falk et al., 2012; Fraga et al., 2013a,b) for complete details of the EEG amplitude modulation rate-of-change features.

\subsubsection{Feature sets and set combination}

Computed features were grouped into four feature sets: spectral, modulation, coherence (MSC), and phase (phase coherence and phase synchrony). To explore the complementarity of the extracted features, combined feature sets were also investigated. Henceforth, we will refer to the "All" feature set as the set that combines all the extracted features and the "Spec-Mod" set as the set that combines the spectral and amplitude-modulation based features. This latter combined set is motivated by the recent results suggesting the complementary of the two feature domains for AD characterization (Fraga et al., 2013a).

\subsubsection{Epoch averaging in the feature domain}

As an additional EEG "cleaning" tool, we use epoch averaging in the feature domain as a way of improving the signal-to-noise ratio (SNR) of the extracted features. This procedure was recently shown to improve the clustering of amplitude modulation rateof-change features, thus leading to higher diagnostic accuracies (Fraga et al., 2013b). This procedure is akin to the epoch averaging step commonly performed in event related potential studies 
(Luck, 2005), but differs in the sense that it is performed in the (non-linear) feature domain and not in the time domain. In our experiments, averaging is performed over features extracted from five consecutive epochs, as motivated by Fraga et al. (2013b).

\subsection{AUTOMATED SALIENT FEATURE SELECTION AND AD CLASSIFICATION}

The machine learning and pattern recognition literature has presented a plethora of possible feature selection and classification algorithms which can be fine-tuned to specific applications and feature sets. For the experiments herein, however, we are interested in understanding the effects of AAR algorithms on different EEG feature sets and on overall diagnostic performance, and not the effects of different selection/classification algorithms and their internal parameters. As such, our experiments are based on a support vector machine (SVM) feature selection and classification algorithm that is widely used in the EEG-based AD diagnosis literature (Lehmann et al., 2007; Trambaiolli et al., 2011a; Falk et al., 2012; Fraga et al., 2013b). The open-source Weka SVM implementation was used in our experiments; default parameters included a polynomial kernel, regularization coefficient $C=$ 1 , and hyperplane shaping coefficient $\gamma=0.01$. A description of the SVM-based feature selection and classification algorithm is beyond the scope of this paper, and the interested reader is referred to (e.g., Cristianini and Shawe-Taylor, 2000; Hall et al., 2009) for more details.

In our experiments, $25 \%$ of the available data was randomly set aside for feature selection and the remaining $75 \%$ was used for classifier training/testing using 10-fold cross validation. Using disjoint sets for feature selection and classifier training reduces any unwanted biases in the reported performance figures. To remain inline with the existing EEG-based AD diagnostic literature, feature selection was used to sift out the 24 most relevant features for $\mathrm{AD}$ diagnosis. In this study, we investigate the effects of AAR on AD diagnostic performance using three classification tasks, namely, (a) Task 1: $N$ vs. $A D 1$ vs. $A D 2$; (b) Task 2: $N$ vs. $A D 1$; and (c) Task 3: $A D 1$ vs. $A D 2$. The first task explores the impact of AAR on a more challenging 3-class problem discriminating between mild- $\mathrm{AD}$, moderate- $\mathrm{AD}$, and healthy controls. The second, in turn, explores the impact on discrimination capabilities between healthy aging and mild-AD, thus exemplifies the case of early detection. Lastly, the third assesses the impact of AAR on EEG-based disease progression monitoring (i.e., from mild to moderate).

\subsection{PERFORMANCE METRICS AND THE "GOLD STANDARD" SYSTEM}

In order to assess diagnosis performance, classification accuracy is used as a performance metric. Moreover, for the two 2-class problems described above, diagnosis sensitivity and specificity are also used. Throughout the remainder of this paper we will assess the impact of AAR on AD classification by measuring the performance gains obtained relative to the baseline (i.e., using the "raw" EEG data). The relative performance gain is given by:

$$
\text { Gain }=\frac{\operatorname{Perf}_{\text {AAR }}-\text { Perf }_{\text {base }}}{\text { Perf }_{\text {base }}} \times 100 \% \text {, }
$$

where "Perf $f_{A A R}$ " and "Perf $f_{\text {base }}$ " refer to the obtained performances (i.e., accuracy, sensitivity, or specificity) after artifact removal and before, respectively. For comparison purposes, we use a so-called gold-standard system to benchmark the results; the system is based on the manually selected artifact-free EEG dataset and the "All-feature" set with 5-epoch feature averaging. On the 3-class task, the gold standard achieves an accuracy of $83.8 \%$. For the $N$ vs. $A D 1$ and $A D 1$ vs. $A D 2$ tasks, in turn, accuracies of $93.2 \%$ and $92.8 \%$ are obtained, respectively.

\section{EXPERIMENTAL RESULTS}

Table 1 reports the accuracies achieved with the baseline system in the top row, followed by the relative gains (Equation 3) achieved with the different AAR algorithms for the four feature sets and two combined feature sets (i.e., "All" and "Spec-Mod") for the 3-class task. Table 2 presents the accuracy, sensitivity, and specificity of the baseline system for all feature sets for the two 2-class tasks. In turn, Tables 3, 4 report the relative gains for all AAR-feature set combinations for the $N$ vs. $A D 1$ and $A D 1$ vs. $A D 2$ tasks, respectively. Careful analysis of the Tables suggests that for all three tasks, the wICA AAR algorithm combined with the top 24 features selected from the "All-feature" set resulted in the best classification performance. Tables 5, 6 show the top-24 selected features for each of the three tasks, for the wICA-AAR and gold standard scenarios, respectively. Feature names are reported as "ELECTRODE_BAND_FEATURE" where "ELECTRODE" represents either the 10-20 electrode positions (e.g., PZ) or the virtual bipolar signal (e.g., P3-P4), "BAND" represents the EEG frequency band (e.g., delta), and "FEATURE" provides a descriptive indication of the feature representation (e.g., "pwr" corresponds to spectral power; "malpha" to modulation rate; "cohe_mag/pha" to magnitude/phase coherence).

\section{DISCUSSION}

\subsection{SALIENT FEATURES}

The list of top-selected features shown in Table 5, 6 show that power spectral and amplitude modulation features are the most salient. Combined, they correspond to 92,83 , and $79 \%$ of the top24 selected features in Tasks 1-3, respectively, for the wICA-AAR

Table 1 | Baseline accuracy per feature set and relative gains obtained after AAR for the 3-class " $N$ vs. $A D 1$ vs. $A D 2$ " task.

\begin{tabular}{|c|c|c|c|c|c|c|}
\hline \multirow[t]{2}{*}{ AAR } & \multicolumn{6}{|c|}{ Feature sets } \\
\hline & Spectrum & Modulation & Coherence & Phase & All & Spec-mod \\
\hline $\begin{array}{l}\text { Baseline } \\
\text { (\%) }\end{array}$ & 73.2 & 68.4 & 60.1 & 45.7 & 72.3 & 73.5 \\
\hline
\end{tabular}

\begin{tabular}{|c|c|c|c|c|c|c|}
\hline \multicolumn{7}{|c|}{ RELATIVE GAINS } \\
\hline SAR & 1.3 & -3.6 & 0.2 & 1.8 & 2.5 & -0.8 \\
\hline SAR-BSS & -5.9 & -10.6 & -6.2 & -12.2 & -1.0 & -3.7 \\
\hline SAR-wICA & -0.8 & -3.0 & 7.6 & 2.6 & 4.5 & 2.5 \\
\hline BSS & -4.0 & -4.6 & -6.5 & -12.2 & -6.6 & -7.4 \\
\hline wICA & 3.3 & 2.9 & 11.5 & 5.5 & 8.4 & 3.8 \\
\hline
\end{tabular}


Table 2 | Baseline performance values for the two, 2-class tasks.

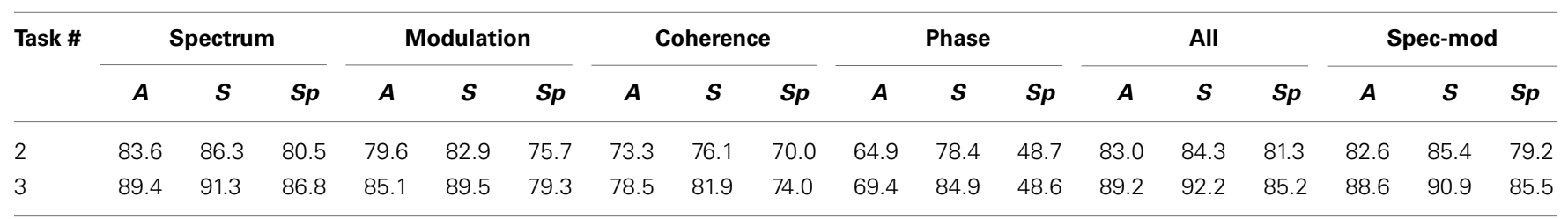

Columns labeled "A, S, and Sp" correspond to accuracy, sensitivity, and specificity, respectively.

Table 3 | Relative gains obtained after AAR for the 2-class " $N$ vs. $A D 1$ " task.

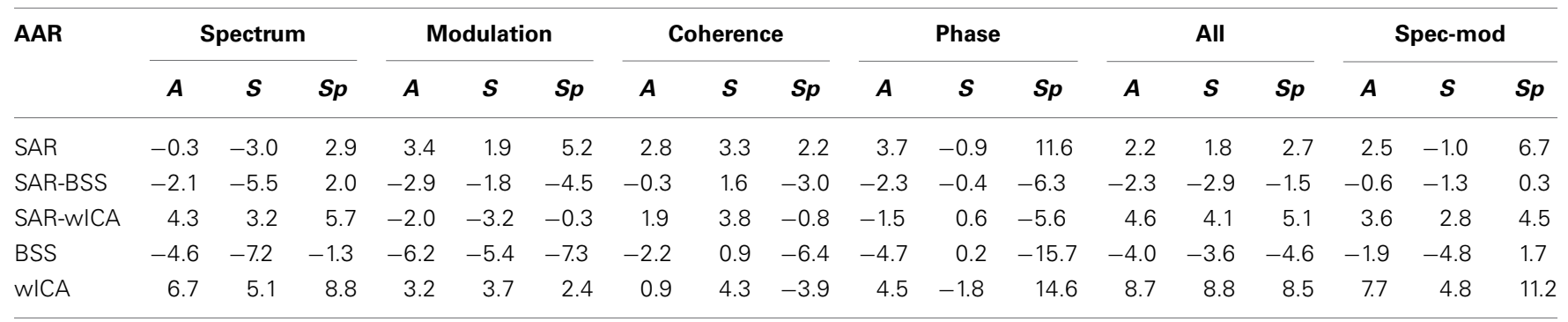

Columns labeled "A, S, and Sp" correspond to accuracy, sensitivity, and specificity, respectively.

Table 4 | Relative gains obtained after AAR for the 2-class "AD1 vs. AD2" task.

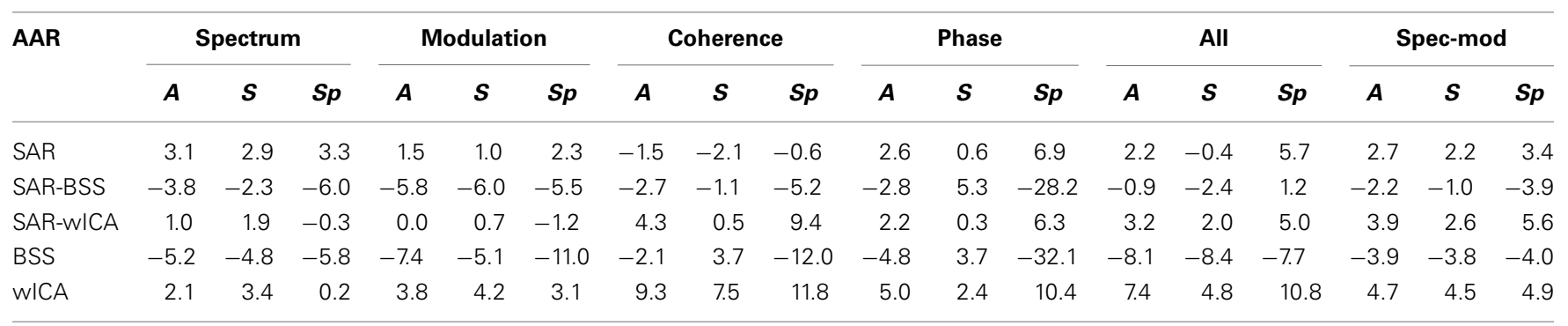

Columns labeled "A, S, and Sp" correspond to accuracy, sensitivity, and specificity, respectively.

scenario. For the gold standard benchmark, such features correspond to 96,79 , and $70 \%$ of the entire feature pool for Tasks 1-3, respectively. This corroborates recent findings showing the complementarity of the two modalities for AD diagnosis (Fraga et al., 2013a). Phase features, in turn, were seldom selected in both the wICA-AAR and gold standard scenarios, thus suggesting they play a small role in EEG-based AD diagnosis. The global field synchrony measure, in fact, did not show up in the top-24 feature subsets for any of the three Tasks.

Moreover, when discriminating between the three classes, features from the temporal and parietal regions showed to be important across the two scenarios. For the $N$ vs. $A D 1$ task, in turn, frontal and temporal regions stood out. For Task 3, features from the temporal and frontal regions were most salient for the wICA-AAR scenario, whereas the temporal and parietal regions stood out for the gold standard. Frontal region data may be corrupted by eye blinks/movement artifacts, thus are likely rejected by human experts. By automatically removing the artifacts from the data, useful discriminatory information may remain in such electrodes, thus assisting in $\mathrm{AD}$ diagnosis.

As for frequency bands, in the wICA scenario, delta and beta band features corresponded to roughly $70 \%$ of the selected features for each of the three tasks, followed by alpha band features
(15\%), thus corroborating previous studies that show the slowing of the EEG with AD (e.g., Coben et al., 1983; Elmståhl et al., 1994; Sankari et al., 2012; Waser et al., 2013). In the gold standard scenario, the delta, theta and beta features were most prevalent, amounting to about $80 \%$ of the selected features. Theta band features were particularly useful for Task 3, a finding previously reported in the $\mathrm{AD}$ severity monitoring literature (Coben et al., 1985). It is important to emphasize that none of the features extracted from the gamma bands were selected. It is hypothesized that this may be due to the fact that such higher frequencies are most sensitive to EEG artifacts, thus are (i) often discarded by human experts and (ii) may be severely distorted by the enhancement algorithms to a point of removing any existing discriminatory information. Lastly, it was observed that of the 24 selected features, roughly $40 \%$ corresponded to information extracted from interhemispheric/virtual bipolar signals, thus corroborating evidence of an interhemispheric disconnection with AD (Jeong, 2004).

\subsection{EFFECTS OF AAR ON FEATURE DISTRIBUTIONS}

In order to characterize the effects of the wICA algorithm on the distribution and statistics of the salient features, we utilize a socalled distribution overlap metric which measures the amount 
Table 5 | Selected features used with the wICA-AAR automated system.

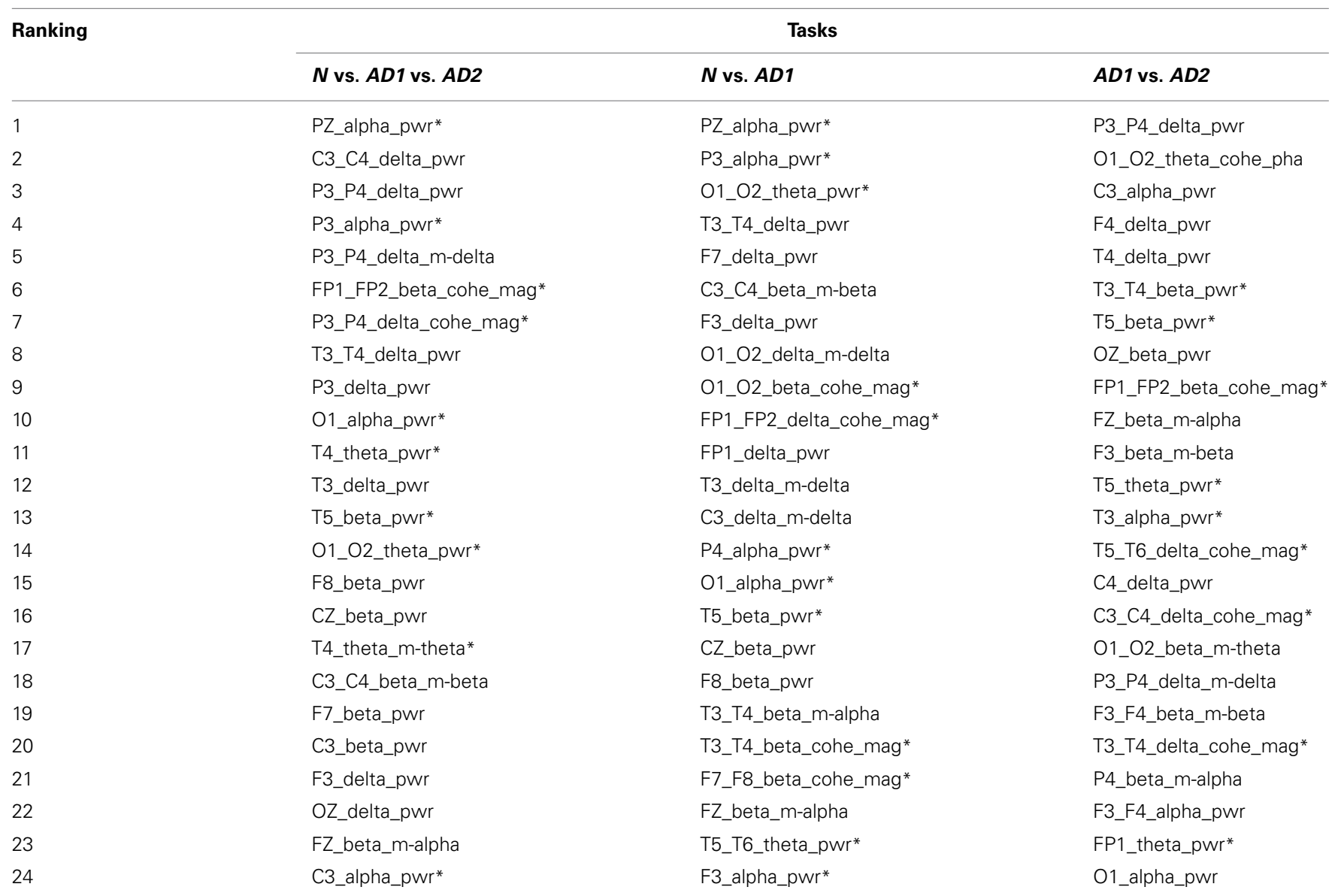

\section{NUMBER OF FEATURES PER FEATURE SET}

$\begin{array}{lccc}\text { Spectral power } & 18(7) & 14(8) & 13(5) \\ \text { Modulation } & 4(1) & 6(0) & 6(0) \\ \text { Coherence } & 2(2) & 4(4) & 4(4) \\ \text { Phase } & 0(0) & 0(0) & 1(0)\end{array}$

\section{NUMBER OF FEATURES PER BRAIN REGION}

$\begin{array}{llrr}\text { Frontal } & 5(1) & 8(3) & 7(2) \\ \text { Central } & 5(1) & 3(0) & 3(1) \\ \text { Temporal } & 5(3) & 6(3) & 7(6) \\ \text { Parietal } & 6(3) & 3(3) & 3(0) \\ \text { Occipital } & 3(2) & 4(3) & 4(0)\end{array}$

\section{NUMBER OF FEATURES PER FREQUENCY BAND}

$\begin{array}{llll}\text { Delta } & 9(1) & 8(1) & 8(3) \\ \text { Theta } & 3(3) & 2(2) & 3(2) \\ \text { Alpha } & 4(4) & 5(5) & 4(1) \\ \text { Beta } & 8(2) & 9(4) & 9(3)\end{array}$

\section{NUMBER OF FEATURES FROM VIRTUAL CHANNELS}

Interhemispheric

8 (3)

9 (6)

$11(5)$

Features with an asterisk represent those with an overlap in histograms between pre- and post-AAR $\geq 80 \%$. Last four sections show, from top to bottom, the number of features that belong to each of the four feature sets, brain regions, frequency band, and montage, respectively. Values reported between parentheses represent those with pre-post AAR histogram overlap $\geq 80 \%$. 
Table 6 | Selected features used with the gold standard system.

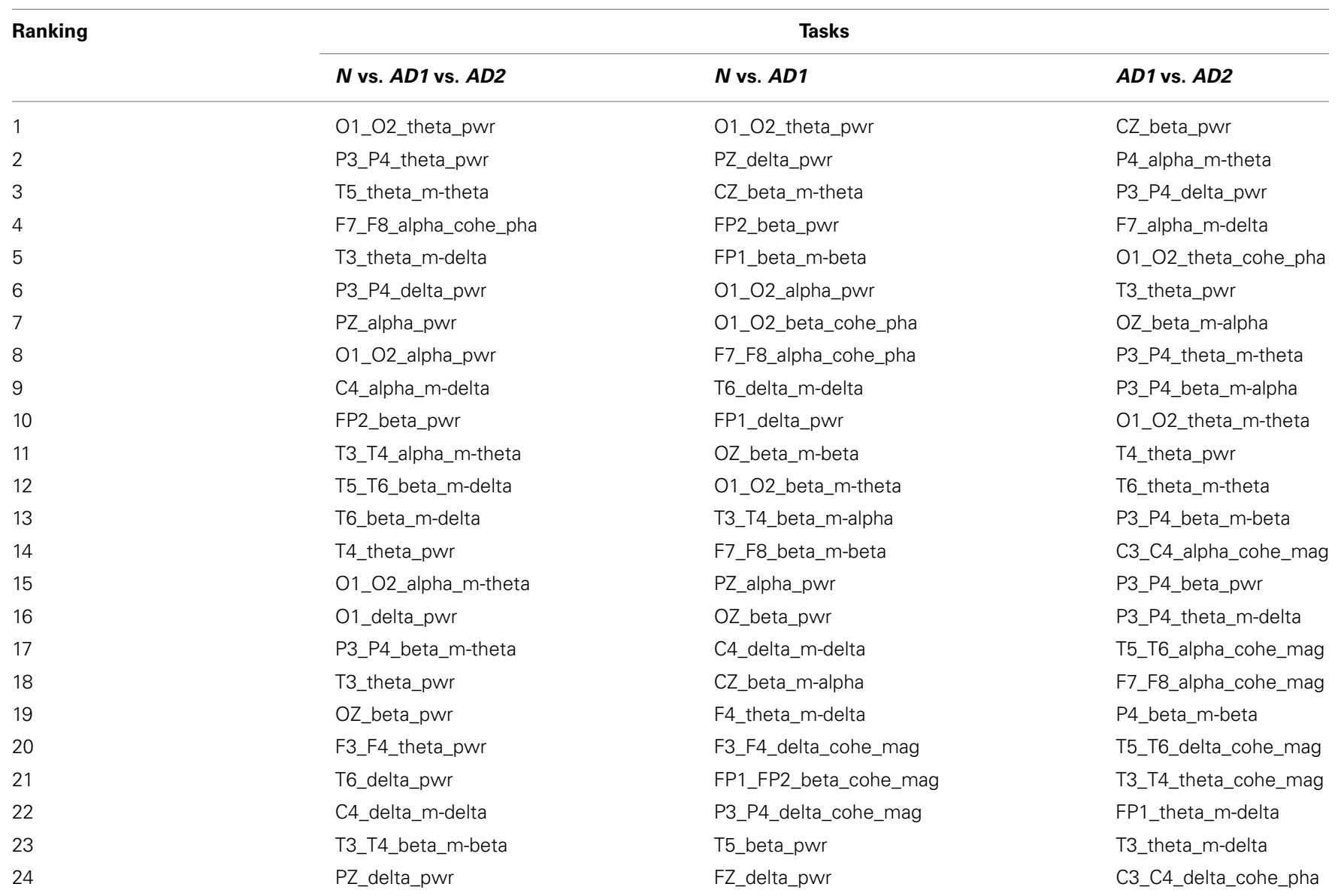

\section{NUMBER OF FEATURES PER FEATURE SET}

Spectral power

Modulation

Coherence

Phase

13

10

0

1
O1_O2_theta_pwr
PZ_delta_pwr
CZ_beta_m-theta
FP2_beta_pwr
FP1_beta_m-beta
O1_O2_alpha_pwr
01_O2_beta_cohe_pha
F7_F8_alpha_cohe_pha
T6_delta_m-delta
FP1_delta_pwr
OZ_beta_m-beta
O1_O2_beta_m-theta
T4_beta_m-alpha
PZ_alpha_pwr
C7 beta m-alpha
4_theta_m-delta
FP1_FP2_beta_cohe_mag
P3_P4_delta_cohe_mag
FZ_delta_pwr

\section{NUMBER OF FEATURES PER BRAIN REGION}

$\begin{array}{lc}\text { Frontal } & 3 \\ \text { Central } & 2 \\ \text { Temporal } & 9 \\ \text { Parietal } & 5 \\ \text { Occipital } & 5\end{array}$

$\begin{array}{cc}9 & 5 \\ 10 & 12 \\ 3 & 12 \\ 2 & 5 \\ & 2\end{array}$

\section{NUMBER OF FEATURES PER FREQUENCY BAND}

$\begin{array}{llcc}\text { Delta } & 5 & 7 & 3 \\ \text { Theta } & 7 & 2 & 10 \\ \text { Alpha } & 6 & 3 & 5 \\ \text { Beta } & 6 & 12 & 6\end{array}$

\section{NUMBER OF FEATURES FROM VIRTUAL CHANNELS}

Interhemispheric

11

10

14

Last four sections show, from top to bottom, the number of features that belong to each of the four feature sets, brain regions, frequency band, and montage, respectively. 
of overlap between the histogram of a particular feature before and after wICA AAR. The metric is normalized to lie between $0-100 \%$ with 0 and $100 \%$ overlap values suggesting complete change and no change in feature statistics post-AAR, respectively. For simplicity, Table 5 highlights features which resulted in an overlap greater than $80 \%$, thus can be considered as irrelevant statistical changes. For illustration purposes, Figure 2 presents the pre- and post-AAR histograms for two features. Subplot (A) is for a feature with an overlap of $83 \%$ (FZ_beta_m-alpha) and subplot (B) for a feature with 49\% overlap (FZ_beta_m-alpha). As can be seen from Table 5, roughly half of the top-24 features did not present relevant modifications in their distributions post wICA-AAR processing. Moreover, coherence features were found to be the least affected, whereas the amplitude modulation ones were most affected. For Tasks 1 and 2, alpha and theta bands features were least affected; however, features from such frequency bands only correspond to roughly $30 \%$ of the top- 24 selected features. Interestingly, features from such band correspond to $55 \%$ and $63 \%$ of the features selected manually for Tasks 1 and 3 , respectively (see Table 6), thus suggesting their robustness to artifacts.

\subsection{AUTOMATED vs. HUMAN EXPERT ARTIFACT REMOVAL}

From Tables 1-4, it can be seen that wICA-AAR combined with classifiers trained on the top-24 features found from the "Allfeatures" pool (see Tables 5, 6) resulted in the best classification performance. For the three-class task, such automated system resulted in an overall classification accuracy of $78.9 \%$, which is significantly higher than chance and inline with what was achieved with the gold standard (i.e., 83.8\%). For Task 2, in turn, accuracy, sensitivity, and specificity of $90.8,92.5$, and $88.8 \%$ could be achieved, respectively with the automated system. This also compares favorably with the gold standard, which attained performance levels of 93.2, 95, and 91\%, respectively. Moreover, the wICA and SAR-wICA combination resulted in substantial improvements for the coherence features, thus corroborating findings from Castellanos and Makarov (2006).
Interestingly, for Task 3 involving $\mathrm{AD} 1$ and $\mathrm{AD} 2$ patients, the wICA-AAR system outperformed the gold standard, achieving accuracy, sensitivity, and specificity values of 96.3, 96.9, and $95.5 \%$, respectively. The gold standard, in turn, obtained values $92.8,97.3$, and $86.7 \%$, respectively. It is suspected that this improved performance was obtained due to information harnessed from the frontal electrodes, which were often selected by the wICA-processed data and not from the manually-selected data. Frontal electrodes are susceptible to eye-related artifacts and are likely often discarded by human experts. Notwithstanding, the frontal region has been shown in classical studies to be severely affected by disease progression (Mann et al., 1988; DeKosky and Scheff, 1990). These findings show the relevance of an automated system in assisting clinicians with diagnosis.

Moreover, from Tables 1-4 it can be seen that the BSS algorithm and its combination with SAR resulted in performance decreases relative to the baseline system trained on raw noisy data for all tested feature sets and tasks. This suggests that while BSS can be used to reliably remove ocular artifacts (Gómez-Herrero et al., 2006), its processing also removes important discriminatory information from the raw EEG data. Hence, it is suggested that BSS be avoided in EEG-based AD diagnosis systems.

Lastly, we explored the gains obtained with feature averaging as a simple SNR improvement tool. For Task 1, the accuracy gains relative to the baseline obtained with only feature averaging (i.e., raw EEG data without AAR) were of 3.3, 4.9, 3.4, and $1.9 \%$ for the spectral, amplitude modulation, coherence, and phase feature sets, respectively. For Task 2, in turn, these relative accuracy gains were of $1.5,1.1,2.6,2.2 \%$, respectively. Lastly, for Task 3 the relative gains were $3,0.8,2.4$, and $2 \%$ respectively. As can be seen, simple feature averaging (Fraga et al., 2013b) can be used as an effective tool that can be combined with AAR algorithms to further improve diagnostic performance.

\subsection{LIMITATIONS}

The three enhancement algorithms explored here represented the state-of-the-art applicable to the constraints imposed by
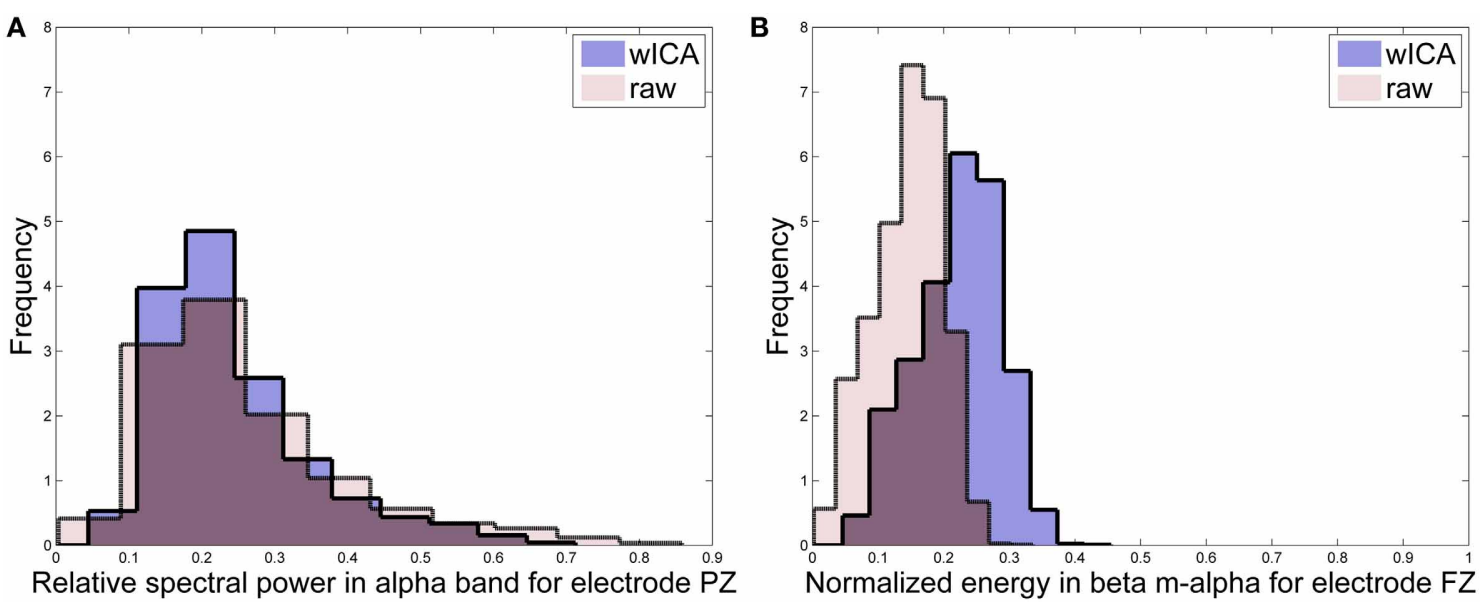

FIGURE 2 | Histograms for features (A) PZ_alpha_pwr and (B) FZ_beta_m-alpha. 
our available database, such as small number of channels (20), limited amount of data per participant, and lack of EOG reference channels. For future studies without these limitations, alternate AAR algorithms can be explored. For example, for studies involving EEG with over 64 channels and EOG, the ADJUST (Automatic EEG artifact Detection based on the Joint Use of Spatial and Temporal features) (Mognon et al., 2011) and FASTER (Fully Automated Statistical Thresholding for EEG artifact Rejection) (Nolan et al., 2010) algorithms can be used. On our 20-channel dataset, we found the use of these two algorithms to lead to over rejection of components deemed artifactual, thus negatively impacting diagnostic performance. Alternately, if larger amounts of EEG data are collected per participant, other data-driven methods may be used, such as the weighted support vector machine-based AAR method proposed by Shao et al. (2009). Lastly, if auxiliary signals are recorded simultaneously with EEG data, other multi-channel AAR methods may be applied. Representative examples include the use of EOG or signals from optical eye tracking systems to develop adaptive filtering schemes (e.g., Joyce et al., 2004; Schlögl et al., 2007; Samadi and Cooke, 2013), or even the use of gyroscopes in ambulatory EEG systems to flag EEG segments collected during head movements (ORegan and Marnane, 2013).

\section{CONCLUSION}

The last decade has seen a rise in the development of EEGbased tools to assist clinicians with AD diagnosis. This paper has evaluated the effects of different state-of-the-art AAR algorithms on diagnosis performance; AAR algorithms were tested both alone and in tandem. Experimental results showed the wavelet enhanced ICA (wICA) AAR algorithm outperforming all other algorithms across four investigated feature sets (spectral, amplitude modulate rate-of-change, coherence, phase), as well as two combined feature sets ("All" and "Spectral-modulation"). In a disease progression monitoring task (Task 3), the automated system was shown to outperform a diagnostic system trained on artifact-free data processed by human experts. Such findings suggest that the discard of useful discriminatory information can be avoided if AAR algorithms are used. Ultimately, it is hoped that such fully-automated diagnostic tools be used to assist clinicians not only with early diagnostics, but also with disease progression monitoring and assessment.

\section{ACKNOWLEDGMENTS}

This work was funded by the Natural Sciences and Engineering Research Council of Canada (NSERC) and the Foundation for Research Support of the State of São Paulo (FAPESP). The authors would also like to thank Dr. Justin Dauwels for sharing his scripts to calculate the global field synchrony feature.

\section{REFERENCES}

Achanccaray, D. R., and Meggiolaro, M. A. (2008). "Detection of artifacts from EEG data using wavelet transform, high-order statistics and neural networks," in XVII Brazilian Conference on Automatica (Juiz de Fora).

Adeli, H., Ghosh-Dastidar, S., and Dadmehr, N. (2005). Alzheimer's disease: models of computation and analysis of EEGs. Clin. EEG Neurosci. 36, 131-140. doi: $10.1177 / 155005940503600303$

Adler, G., Brassen, S., and Jajcevic, A. (2003). EEG coherence in Alzheimer's dementia. J. Neural Transm. 110, 1051-1058. doi: 10.1007/s00702-003-0024-8
Akhtar, M. T., Mitsuhashi, W., and James, C. J. (2012). Employing spatially constrained ICA and wavelet denoising for automatic removal of artifacts from multichannel EEG data. Signal Process. 92, 401-416. doi: 10.1016/j.sigpro.2011.08.005

Alzheimer Association. (2013). 2013 Alzheimer's disease facts and figures. J. Alzheimer's Assoc. 9, 208-245. doi: 10.1016/j.jalz.2013.02.003

Atlas, L., and Shamma, S. A. (2003). Joint acoustic and modulation frequency. EURASIP J. Appl. Signal Process. 2003, 668-675. doi: 10.1155/S1110865703305013

Babiloni, C., Lizio, R., Vecchio, F., Frisoni, G. B., Pievani, M., Geroldi, C., et al. (2010). Reactivity of cortical alpha rhythms to eye opening in mild cognitive impairment and Alzheimer's disease: an EEG study. J. Alzheimer's Dis. 22, 1047-1064. doi: 10.3233/JAD-2010-100798

Bedrosian, E. (1963). A product theorem for Hilbert transforms. Proc. IEEE 51, 868-869. doi: 10.1109/PROC.1963.2308

Belouchrani, A., Abed-Meraim, K., Cardoso, J.-F., and Moulines, E. (1997). A blind source separation technique using second-order statistics. IEEE Trans. Signal Process. 45, 434-444. doi: 10.1109/78.554307

Besthorn, C., Förstl, H., Geiger-Kabisch, C., Sattel, H., Gasser, T., and SchreiterGasser, U. (1994). EEG coherence in alzheimer disease. Electroencephalogr. Clin. Neurophysiol. 90, 242-245. doi: 10.1016/0013-4694(94)90095-7

Brenner, R. P., Ulrich, R. F., Spiker, D. G., Sclabassi, R. J., Reynolds III, C. F., Marin, R. S., et al. (1986). Computerized EEG spectral analysis in elderly normal, demented and depressed subjects. Electroencephalogr. Clin. Neurophysiol. 64, 483-492. doi: 10.1016/0013-4694(86)90184-7

Brucki, S., Nitrini, R., Caramelli, P., Bertolucci, P. H., and Okamoto, I. H. (2003). Suggestions for utilization of the mini-mental state examination in Brazil. Arq. Neuropsiquiatr. 61, 777-781. doi: 10.1590/S0004-282X2003000500014

Castellanos, N. P., and Makarov, V. A. (2006). Recovering EEG brain signals: artifact suppression with wavelet enhanced independent component analysis. J. Neurosci. Methods 158, 300-312. doi: 10.1016/j.jneumeth.2006. 05.033

Coben, L. A., Danziger, W. L., and Berg, L. (1983). Frequency analysis of the resting awake EEG in mild senile dementia of Alzheimer type. Electroencephalogr. Clin. Neurophysiol. 55, 372-380. doi: 10.1016/0013-4694(83)90124-4

Coben, L. A., Danziger, W., and Storandt, M. (1985). A longitudinal EEG study of mild senile dementia of Alzheimer type: changes at 1 year and at 2.5 years. Electroencephalogr. Clin. Neurophysiol. 61, 101-112. doi: 10.1016/00134694(85) $91048-\mathrm{X}$

Cristianini, N., and Shawe-Taylor, J. (2000). An Introduction to Support Vector Machines and Other Kernel-Based Learning Methods. Cambridge, UK: Cambridge University Press.

Czigler, B., Csikós, D., Hidasi, Z., Anna Gaál, Z., Csibri, É., Kiss, É., et al. (2008). Quantitative EEG in early Alzheimer's disease patients-power spectrum and complexity features. Int. J. Psychophysiol. 68, 75-80. doi: 10.1016/j.ijpsycho.2007.11.002

Daly, I., Nicolaou, N., Nasuto, S. J., and Warwick, K. (2013). Automated artifact removal from the electroencephalogram: a comparative study. Clin. EEG Neurosci. 44, 291-306. doi: 10.1177/1550059413476485

Dauwels, J., Srinivasan, K., Ramasubba Reddy, M., Musha, T., Vialatte, F.-B., Latchoumane, C., et al. (2011). Slowing and loss of complexity in Alzheimer's EEG: two sides of the same coin? Int. J. Alzheimer's Dis. 2011:539621. doi: $10.4061 / 2011 / 539621$

De Clercq, W., Vergult, A., Vanrumste, B., Van Paesschen, W., and Van Huffel, S. (2006). Canonical correlation analysis applied to remove muscle artifacts from the electroencephalogram. IEEE Trans. Biomed. Eng. 53, 2583-2587. doi: 10.1109/TBME.2006.879459

DeKosky, S. T., and Scheff, S. W. (1990). Synapse loss in frontal cortex biopsies in Alzheimer's disease: correlation with cognitive severity. Ann. Neurol. 27, 457464. doi: 10.1002/ana.410270502

Delorme, A., and Makeig, S. (2004). EEGLAB: an open source toolbox for analysis of single-trial EEG dynamics including independent component analysis. J. Neurosci. Methods 134, 9-21. doi: 10.1016/j.jneumeth.2003.10.009

Delorme, A., Sejnowski, T., and Makeig, S. (2007). Enhanced detection of artifacts in EEG data using higher-order statistics and independent component analysis. Neuroimage 34, 1443-1449. doi: 10.1016/j.neuroimage.2006.11.004

Dunkin, J. J., Leuchter, A. F., Newton, T. F., and Cook, I. A. (1994). Reduced EEG coherence in dementia: state or trait marker? Biol. Psychiatry 35, 870-879. doi: 10.1016/0006-3223(94)90023-X 
Elmståhl, S., Rosén, I., and Gullberg, B. (1994). Quantitative EEG in elderly patients with Alzheimer's disease and healthy controls. Dement. Geriatr. Cogn. Disord. 5, 119-124. doi: 10.1159/000106706

Falk, T. H. and Chan, W.-Y. (2008). Modulation filtering for heart and lung sound separation from breath sound recordings. Conf. Proc. IEEE Eng. Med. Biol. Soc. 2008, 1859-1862. doi: 10.1109/IEMBS.2008.4649547

Falk, T. H., Chan, W.-Y., Sejdic, E., and Chau, T. (2010). "Spectro-temporal analysis of auscultatory sounds," in New Developments in Biomedical Engineering, ed D. Campolo (Rijeka: In-Tech Publishing), 93-104.

Falk, T. H., Fraga, F. J., Trambaiolli, L., and Anghinah, R. (2012). EEG amplitude modulation analysis for semi-automated diagnosis of Alzheimer's disease. EURASIP J. Adv. Signal Process. 2012, 1-9. doi: 10.1186/1687-6180-2012-192

Fraga, F. J., Falk, T. H., Kanda, P. A. M., and Anghinah, R. (2013a). Characterizing Alzheimer's disease severity via resting-awake EEG amplitude modulation analysis. PLoS ONE 8:e72240. doi: 10.1371/journal.pone.0072240

Fraga, F. J., Falk, T. H., Trambaiolli, L. R., Oliveira, E. F., Pinaya, W. H., Kanda, P. A., et al. (2013b). "Towards an EEG-based biomarker for Alzheimer's disease: improving amplitude modulation analysis features," in Proceeding IEEE International Conference on Acoustics, Speech and Signal Processing (Vancouver, BC), 1207-1211.

Giaquinto, S., and Nolfe, G. (1986). The EEG in the normal elderly: a contribution to the interpretation of aging and dementia. Electroencephalogr. Clin. Neurophysiol. 63, 540-546. doi: 10.1016/0013-4694(86)90141-0

Gómez-Herrero, G. (2007). Automatic Artifact Removal (AAR) Toolbox v1. 3 (Release 09.12. 2007) for MATLAB

Gómez-Herrero, G., De Clercq, W., Anwar, H., Kara, O., Egiazarian, K., Van Huffel, S., et al. (2006). "Automatic removal of ocular artifacts in the EEG without an EOG reference channel," in Proceedings Nordic Signal Processing Symposium (Rejkjavik), 130-133.

Hall, M., Frank, E., Holmes, G., Pfahringer, B., Reutemann, P., and Witten, I. H. (2009). The weka data mining software: an update. ACM SIGKDD Explor. Newslett. 11, 10-18. doi: 10.1145/1656274.1656278

James, C. J., and Hesse, C. W. (2005). Independent component analysis for biomedical signals. Physiol. Meas. 26, R15. doi: 10.1088/0967-3334/26/1/R02

Jeong, J. (2004). EEG dynamics in patients with Alzheimer's disease. Clin. Neurophysiol. 115, 1490-1505. doi: 10.1016/j.clinph.2004.01.001

Joyce, C. A., Gorodnitsky, I. F., and Kutas, M. (2004). Automatic removal of eye movement and blink artifacts from EEG data using blind component separation. Psychophysiology 41, 313-325. doi: 10.1111/j.1469-8986.2003.00141.x

Jung, T.-P., Makeig, S., Humphries, C., Lee, T.-W., Mckeown, M. J., Iragui, V., et al. (2000). Removing electroencephalographic artifacts by blind source separation. Psychophysiology 37, 163-178. doi: 10.1111/1469-8986.3720163

Kanda, P. A. M., Trambaiolli, L. R., Lorena, A. C., Fraga, F. J., Basile, L. F. I., Nitrini, R., et al. (2013). Clinician's road map to wavelet EEG as an Alzheimer's disease biomarker. Clin. EEG Neurosci. doi: 10.1177/1550059413486272. [Epub ahead of print].

Knott, V., Mohr, E., Mahoney, C., and Ilivitsky, V. (2000). Electroencephalographic coherence in Alzheimer's disease: comparisons with a control group and population norms. J. Geriatr. Psychiatr. Neurol. 13, 1-8. doi: 10.1177/089198870001300101

Koenig, T., Lehmann, D., Saito, N., Kuginuki, T., Kinoshita, T., and Koukkou, M. (2001). Decreased functional connectivity of EEG theta-frequency activity in first-episode, neuroleptic-nave patients with schizophrenia: preliminary results. Schizophr. Res. 50, 55-60. doi: 10.1016/S0920-9964 (00)00154-7

Koenig, T., Prichep, L., Dierks, T., Hubl, D., Wahlund, L., John, E., et al. (2005). Decreased EEG synchronization in Alzheimer's disease and mild cognitive impairment. Neurobiol. Aging 26, 165-171. doi: 10.1016/j.neurobiolaging.2004.03.008

Krishnaveni, V., Jayaraman, S., Anitha, L., and Ramadoss, K. (2006). Removal of ocular artifacts from EEG using adaptive thresholding of wavelet coefficients. J. Neural Eng. 3, 338. doi: 10.1088/1741-2560/3/4/011

Labate, D., La Foresta, F., Inuso, G., and Morabito, F. C. (2011). "Remarks about wavelet analysis in the EEG artifacts detection," in Proceedings of the Italian Workshop on Neural Nets (Vietri sul Mare: IOS Press), 99-106.

Laxton, A. W., Tang-Wai, D. F., McAndrews, M. P., Zumsteg, D., Wennberg, R., Keren, R., et al. (2010). A phase I trial of deep brain stimulation of memory circuits in Alzheimer's disease. Ann. Neurol. 68, 521-534. doi: 10.1002/ana.22089

Lehmann, C., Koenig, T., Jelic, V., Prichep, L., John, R. E., Wahlund, L.-O., et al. (2007). Application and comparison of classification algorithms for recognition of Alzheimer's disease in electrical brain activity (EEG). J. Neurosci. Methods 161, 342-350. doi: 10.1016/j.jneumeth.2006.10.023

Leifer, B. P. (2003). Early diagnosis of Alzheimer's disease: clinical and economic benefits. J. Am. Geriatr. Soc. 51, S281-S288. doi: 10.1046/j.1532-5415.5153.x

Leuchter, A. F., Spar, J. E., Walter, D. O., and Weiner, H. (1987) Electroencephalographic spectra and coherence in the diagnosis of Alzheimer'stype and multi-infarct dementia: a pilot study. Arch. Gen. Psychiatry 44, 993. doi: 10.1001/archpsyc.1987.01800230073012

Locatelli, T., Cursi, M., Liberati, D., Franceschi, M., and Comi, G. (1998). EEG coherence in Alzheimer's disease. Electroencephalogr. Clin. Neurophysiol. 106, 229-237. doi: 10.1016/S0013-4694(97)00129-6

Luck, S. J. (2005). An Introduction to the Event-Related Potential Technique. Cambridge, MA: A Bradford Book.

Makarov, V. A. (2012). Wavelet Enhanced Independent Component Analysis (wICA) Package For MATLAB.

Malyska, N., Quatieri, T. F., and Sturim, D. (2005). "Automatic dysphonia recognition using biologically inspired amplitude-modulation features," in Proceedings ICASSP, Vol. 1 (Philadelphia, PA), 873-876.

Mann, D., Marcyniuk, B., Yates, P., Neary, D., and Snowden, J. (1988). The progression of the pathological changes of Alzheimer's disease in frontal and temporal neocortex examined both at biopsy and at autopsy. Neuropathol. Appl. Neurobiol. 14, 177-195. doi: 10.1111/j.1365-2990.1988.tb00880.x

McKhann, G., Drachman, D., Folstein, M., Katzman, R., Price, D., and Stadlan, E. M. (1984). Clinical diagnosis of Alzheimer's disease report of the NINCDSADRDA work group under the auspices of department of health and human services task force on Alzheimer's disease. Neurology 34, 939-939. doi: 10.1212/WNL.34.7.939

Mognon, A., Jovicich, J., Bruzzone, L., and Buiatti, M. (2011). Adjust: an automatic EEG artifact detector based on the joint use of spatial and temporal features. Psychophysiology 48, 229-240. doi: 10.1111/j.1469-8986.2010.01061.x

Moore, C. I., and Cao, R. (2008). The hemo-neural hypothesis: on the role of blood flow in information processing. J. Neurophysiol. 99, 2035-2047. doi: 10.1152/jn.01366.2006

Moretti, D., Fracassi, C., Pievani, M., Geroldi, C., Binetti, G., Zanetti, O., et al. (2009). Increase of theta/gamma ratio is associated with memory impairment. Clin. Neurophysiol. 120, 295-303. doi: 10.1016/j.clinph.2008.11.012

Moretti, D., Frisoni, G., Fracassi, C., Pievani, M., Geroldi, C., Binetti, G., et al. (2011). MCI patients' EEGs show group differences between those who progress and those who do not progress to AD. Neurobiol. Aging 32, 563-571. doi: 10.1016/j.neurobiolaging.2009.04.003

Moretti, D., Prestia, A., Fracassi, C., Binetti, G., Zanetti, O., and Frisoni, G. (2012). Specific EEG changes associated with atrophy of hippocampus in subjects with mild cognitive impairment and Alzheimer's disease. Int. J. Alzheimer's Dis 2012:253153. doi: 10.1155/2012/253153

Nolan, H., Whelan, R., and Reilly, R. (2010). FASTER: Fully automated statistical thresholding for EEG artifact rejection. J. Neurosci. Methods 192, 152-162. doi: 10.1016/j.jneumeth.2010.07.015

Nolte, G., Bai, O., Wheaton, L., Mari, Z., Vorbach, S., and Hallett, M. (2004). Identifying true brain interaction from EEG data using the imaginary part of coherency. Clin. Neurophysiol. 115, 2292-2307. doi: 10.1016/j.clinph.2004.04.029

Nunez, P. L. (2006). Electric Fields of the Brain: the Neurophysics of EEG. New York, NY: Oxford University Press. doi: 10.1093/acprof:oso/9780195050387.001.0001

ORegan, S., and Marnane, W. (2013). Multimodal detection of headmovement artefacts in eeg. J. Neurosci. Methods 218, 110-120. doi: 10.1016/j.jneumeth.2013.04.017

Park, Y.-M., Che, H.-J., Im, C.-H., Jung, H.-T., Bae, S.-M., and Lee, S.H. (2008). Decreased EEG synchronization and its correlation with symptom severity in Alzheimer's disease. Neurosci. Res. 62, 112-117. doi: 10.1016/j.neures.2008.06.009

Penttilä, M., Partanen, J. V., Soininen, H., and Riekkinen, P. (1985). Quantitative analysis of occipital EEG in different stages of Alzheimer's disease. Electroencephalogr. Clin. Neurophysiol. 60, 1-6. doi: 10.1016/0013-4694(85)90942-3

Raicher, I., Takahashi, D., Kanda, P., Nitrini, R., and Anghinah, R. (2008). qEEG spectral peak in Alzheimer's disease. Dement. Neuropsychol. 2, 9-12.

Romero, S., Mañanas, M. A., and Barbanoj, M. J. (2008). A comparative study of automatic techniques for ocular artifact reduction in spontaneous EEG signals based on clinical target variables: a simulation case. Comp. Biol. Med. 38, 348-360. doi: 10.1016/j.compbiomed.2007.12.001 
Samadi, M. R. H., and Cooke, N. (2013). "A novel approach for adaptive eeg artefact rejection and eog gaze estimation," in HCI International 2013-Posters' Extended Abstracts, ed C. Stephanidis (Las Vegas, NV: Springer), 603-607. doi: 10.1007/978-3-642-39473-7-120

Sankari, Z., Adeli, H., and Adeli, A. (2012). Wavelet coherence model for diagnosis of Alzheimer's disease. Clin. EEG Neurosci. 43, 268-278. doi: $10.1177 / 1550059412444970$

Sarazin, M., de Souza, L. C., Lehéricy, S., and Dubois, B. (2012). Clinical and research diagnostic criteria for Alzheimer's disease. Neuroimaging Clin. N. Am. 22, 23-32. doi: 10.1016/j.nic.2011.11.004

Schlögl, A., Keinrath, C., Zimmermann, D., Scherer, R., Leeb, R., and Pfurtscheller, G. (2007). A fully automated correction method of EOG artifacts in EEG recordings. Clin. Neurophysiol. 118, 98-104. doi: 10.1016/j.clinph.2006.09.003

Shao, S.-Y., Shen, K.-Q., Ong, C. J., Wilder-Smith, E., and Li, X.-P. (2009). Automatic EEG artifact removal: a weighted support vector machine approach with error correction. IEEE Trans. Biomed. Eng. 56, 336-344. doi: 10.1109/TBME.2008.2005969

Sloan, E. P., Fenton, G. W., Kennedy, N. S., and MacLennan, J. M. (1994). Neurophysiology and SPECT cerebral blood flow patterns in dementia. Electroencephalogr. Clin. Neurophysiol. 91, 163-170. doi: 10.1016/00134694(94)90066-3

Soininen, H., Partanen, J., Laulumaa, V., Helkala, E.-L., Laakso, M., and Riekkinen, P. (1989). Longitudinal EEG spectral analysis in early stage of Alzheimer's disease. Electroencephalogr. Clin. Neurophysiol. 72, 290-297. doi: 10.1016/00134694(89)90064-3

Sörnmo, L., and Laguna, P. (2005). Bioelectrical Signal Processing in Cardiac and Neurological Applications. Burlington, MA: Academic Press.

Srinivasan, R., Winter, W. R., Ding, J., and Nunez, P. L. (2007). EEG and MEG coherence: measures of functional connectivity at distinct spatial scales of neocortical dynamics. J. Neurosci. Methods 166, 41-52. doi: 10.1016/j.jneumeth.2007.06.026

Thatcher, R., Krause, P., and Hrybyk, M. (1986). Cortico-cortical associations and EEG coherence: a two-compartmental model. Electroencephalogr. Clin. Neurophysiol. 64, 123-143. doi: 10.1016/0013-4694(86)90107-0

Trambaiolli, L., Lorena, A., Fraga, F., Kanda, P., Nitrini, R., and Anghinah, R. (2011a). Does EEG montage influence Alzheimer's disease electroclinic diagnosis? Int. J. Alzheimer's Dis. 2011:761891. doi: 10.4061/2011/761891
Trambaiolli, L. R., Falk, T. H., Fraga, F. J., Anghinah, R., and Lorena, A. C. (2011b). "EEG spectro-temporal modulation energy: a new feature for automated diagnosis of Alzheimer's disease," in Proceedings of the International Conference of the IEEE Engineering in Medicine and Biology Society (Boston, MA), 3828-3831.

Trambaiolli, L. R., Lorena, A. C., Fraga, F. J., Kanda, P. A., Anghinah, R., and Nitrini, R. (2011c). Improving Alzheimer's disease diagnosis with machine learning techniques. Clin. EEG Neurosci. 42, 160-165. doi: 10.1177/155005941104 200304

Waser, M., Deistler, M., Garn, H., Benke, T., Dal-Bianco, P., Ransmayr, G., et al. (2013). EEG in the diagnostics of Alzheimer's disease. Stat. Papers 54, 1095-1107. doi: 10.1007/s00362-013-0538-6

WHO and Alzheimer's Disease International. (2012). Dementia: A Public Health Priority. Geneva: Technical report, World Health Organization.

Zikov, T., Bibian, S., Dumont, G. A., Huzmezan, M., and Ries, C. (2002). "A wavelet based de-noising technique for ocular artifact correction of the electroencephalogram," in Proceedings of the International Conference of the IEEE Engineering in Medicine and Biology Society, Vol. 1 (Houston, TX), 98-105.

Conflict of Interest Statement: The authors declare that the research was conducted in the absence of any commercial or financial relationships that could be construed as a potential conflict of interest.

Received: 06 December 2013; accepted: 06 March 2014; published online: 25 March 2014.

Citation: Cassani R, Falk TH, Fraga FJ, Kanda PAM and Anghinah R (2014) The effects of automated artifact removal algorithms on electroencephalographybased Alzheimer's disease diagnosis. Front. Aging Neurosci. 6:55. doi: 10.3389/fnagi. 2014.00055

This article was submitted to the journal Frontiers in Aging Neuroscience.

Copyright (c) 2014 Cassani, Falk, Fraga, Kanda and Anghinah. This is an openaccess article distributed under the terms of the Creative Commons Attribution License (CC BY). The use, distribution or reproduction in other forums is permitted, provided the original author(s) or licensor are credited and that the original publication in this journal is cited, in accordance with accepted academic practice. No use, distribution or reproduction is permitted which does not comply with these terms. 